\title{
Adding extra sensitivity to SMART non-linear actuator using sensor fusion
}

\author{
H Montes, L Pedraza, M Armada, and T Akinfiev \\ Automatic Control Department, Industrial Automation Institute, Madrid, Spain \\ R Caballero \\ Universidad Tecnologica de Panama, Republic of Panama
}

\begin{abstract}
The perspective of using humanoid robots in practical applications is attracting an important research effort and the late steps forward in robot technology shows many remarkable achievements where design aspects, control systems and software evolution regarding humanoid machines have been realised. However, and although most of humanoid robots are intended to offer by some means a good degree of autonomy so that they could deploy all their intrinsic capabilities to perform "useful" tasks, the current state of this technology is being still far from allowing to attain such a goal. Among all the concerned features, and aiming to improve humanoid robot overall performance, it is required that they could work for a long time spending minimum energy without losing their kinematical skills. In this direction, a new kind of non-linear actuator, SMART, based on quasi-resonance principle, have been developed by the Industrial Automation Institute to improve the overall performance of biped locomotion. One of the major advantages provided by SMART is their inherent low energy consumption in comparison to classical transmission ratio actuators. However, due to their intrinsically non-linear characteristic, the practical use of these actuators poses some complications, in particular when it is necessary to realise advanced control schemes. To overcome this problem it is demonstrated how force sensing, implemented in one of the four-bar linkages of the mechanism, along with angular position measurement and motor current sensing, provides, throughout a sensor fusion strategy, an extra sensitivity to the non-linear actuator, resulting in an enhanced responsiveness. By using this approach the foreseen theoretical SMART actuator properties are fully demonstrated and experimental results are used to verify the practical advantages of the proposed method.
\end{abstract}

\section{KEYWORDS}

Non-linear actuators, four-bar linkage, force sensor, sensor fusion, power expenditure, biped robots. 


\section{INTRODUCTION}

Traditionally, biped robots have been designed with the support of three types of actuators: electric motors, pneumatic actuators and hydraulic systems. However, the majority of the researchers have a preference on using electric motors for the actuators of the robot joints (Pfeiffer et al., 2000). Nevertheless, driving robots using electrical motors with constant transmission ratios leads generally to high-energy consumption because the overall system is engineered for the worst case loading conditions. This constitutes a specific constraint when dealing with biped robots and results in a limited autono my when the system is to be powered from batteries.

In order to improve efficiency, some authors suggested the use of passive dynamics (McGeer, 1990) in the design of the robots. Other researchers suggested the use of a chain: motor-gearspring (Pratt and Williamson, 1997) so that the energy is stored during some part of the locomotion cycle. Another interesting solution is the use of biology inspired actuators (Mennito and Buehler, 1997; Yamagushi and Takanishi, 1997) by using elastic fibbers or nonlinear spring. These approaches have some advantages, but the implementation of this type of actuators is complex and difficult to model.

On the other hand it has been shown that non-linear transmission ratio actuator could improve mechanical design and decrease energy consumption in many mechanical systems (Van De Straete and Schutter, 1999). Simulation and experimental results with a simplified laboratory prototype of a biped robot have demonstrated that a specially designed non-linear drive, which shares some properties of quasi-resonance drive (SMART: Special Mechatronic Actuator for Robot joinTs) (Akinfiev et al., 2000; Caballero et al., 2002b), has a better performance than a classical constant transmission ratio actuator. Moreover, it has been demonstrated that non-linear transmission is more efficient than classical constant transmission and saving of power expenditure could reach $75 \%$ as compared with classical design (Armada et al., 2003). This is, of course, of major relevance if we are aiming to extend walking robot functioning.

The SMART drive has been implemented by using a four-bar linkage mechanism, where the individual links are of different length (Akinfiev et al., 2000). This four bar linkage is made up of two real bars: rod and crank, and two virtual bars (see Figure 1). This mechanism has good advantage because between the joints surfaces provide (rolling contacts) good conditions for lubrication (Waldron and Kinzel, 1999). In this way, the low friction properties of rolling contacts are exploited to obtain a joint with lower friction and higher load and relative speed capabilities than would be possible with other good kind of joint. 


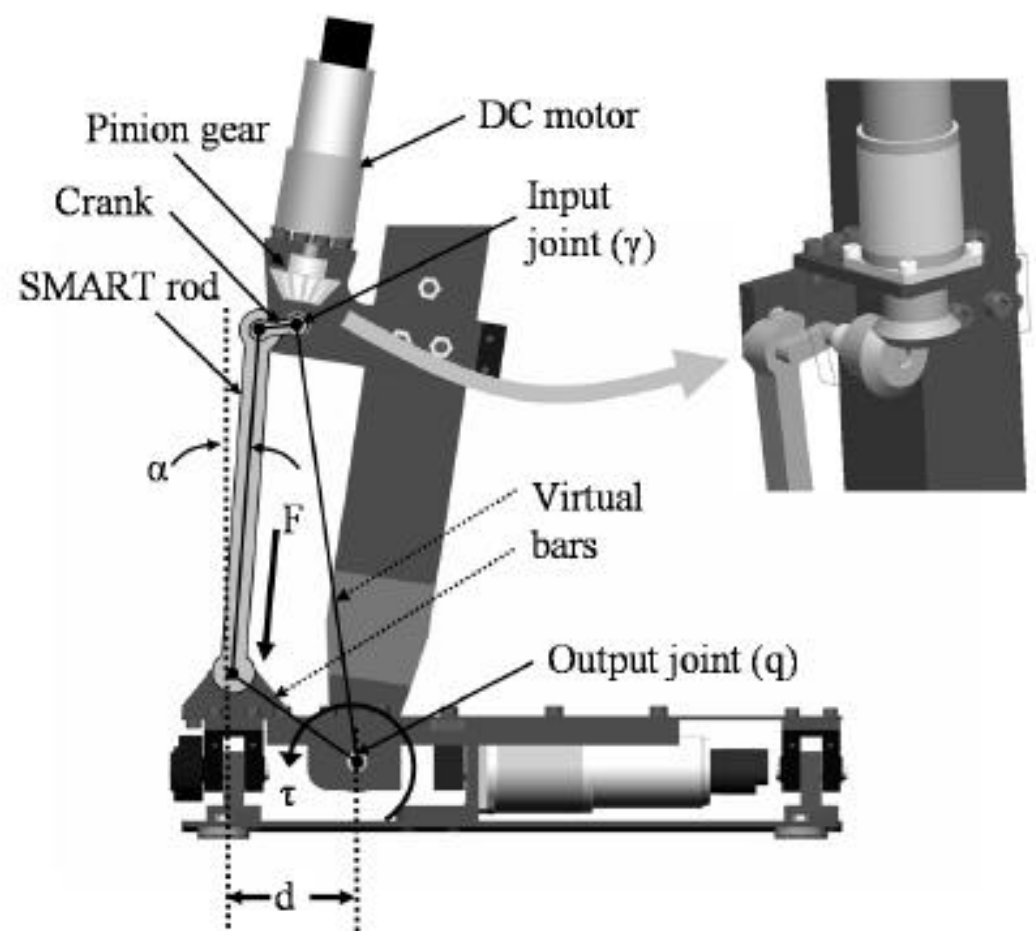

Figure 1 SMART actuator: four-bar linkage and kinematic parameters

With reference to Figure 1, the kinematics parameters are shown, where, the output angle " $q$ " is formed between the vertical axis and the leg; the input angle "?", between the crank prolongation and the perpendicular segment of one virtual bar; the distance " $d$ ", between the ankle joint and the point where the force is applied; and the angle " $a$ ", between the SMART rod and the vertical line. This non-linear actuator, as it will be confirmed later on by the experiments, allows using smaller motors and reduces power expenses without sacrificing output actuator velocity in the centre of the angular range of its trajectory.

The SMART drive kinematics is determined by the next function:

$$
\begin{gathered}
q=2 \arctan \left[\frac{(k 2+k 5 \sin \gamma+k 6 \cos \gamma)-\sqrt{(k 2+k 5 \sin \gamma+k 6 \cos \gamma)^{2}-(k 1-k 3 \cos \gamma)^{2}+(k 7+k 5 \cos \gamma-k 6 \sin \gamma)^{2}}}{(k 1-k 3 \sin \gamma)+(-k 7+k 5 \cos \gamma-k 6 \sin \gamma)}\right]-\delta \\
\alpha=\frac{\pi}{2} 2 \arctan \left[\frac{k 8+\sin \varphi+\sqrt{(k 8+\sin \varphi)^{2}-(k 9-k 10 \cos \varphi-k 11 \sin \varphi)^{2}+(k 12+\cos \varphi)^{2}}}{k 9-k 10 \cos \varphi-k 11 \sin \varphi+k 12+\cos \varphi}\right]
\end{gathered}
$$

Where,

$\gamma: \quad$ Motor axis angle (input angle)

$\varphi=\gamma+0.2846[\mathrm{rad}]$

$k 1 \ldots k 12$ : Constant related with SMART linkage dimension

$q: \quad$ Angle joint output

$\alpha: \quad$ Angle of SMART rod

$\delta=\pi-0.61[\mathrm{rad}]$ 
The SMART actuator is characterised by the change of its reduction ratio from some value in the medium part of the trajectory up to infinitum in its extreme positions (see Figure 2a). With this characteristic such drive minimizes energy expenditure several times and therefore makes obtainable a better overall performance in walking locomotion (Caballero, 2002a). Nevertheless, as it is shown in Figure 2a, the relationship between input joint angle "?" and output joint " $q$ " is non-linear and periodic, and for the same value of " $q$ " there are several possible values of "?" (please notice that Figure 2 shows only half period of the trajectory. For more details, please refer to (Caballero, 2002a)). This means that some "difficulty" is to be paid for the advantages provided by SMART.

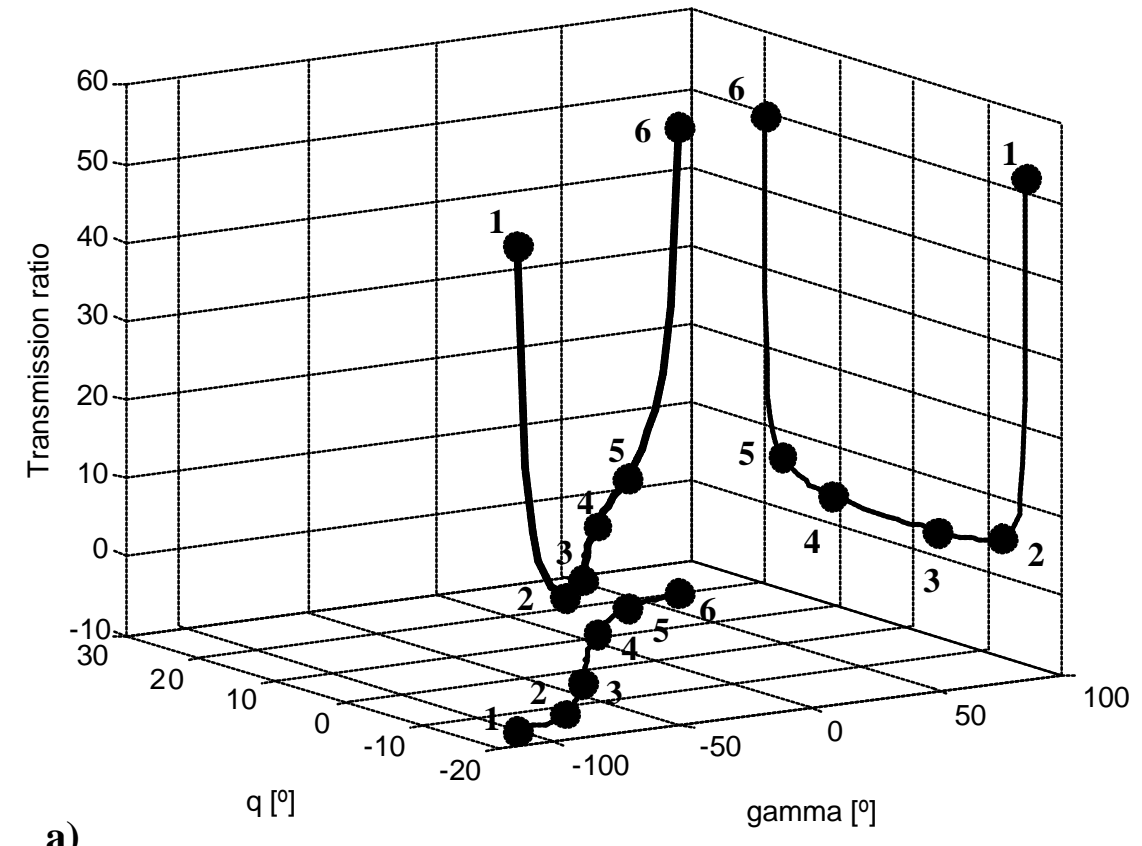

a)
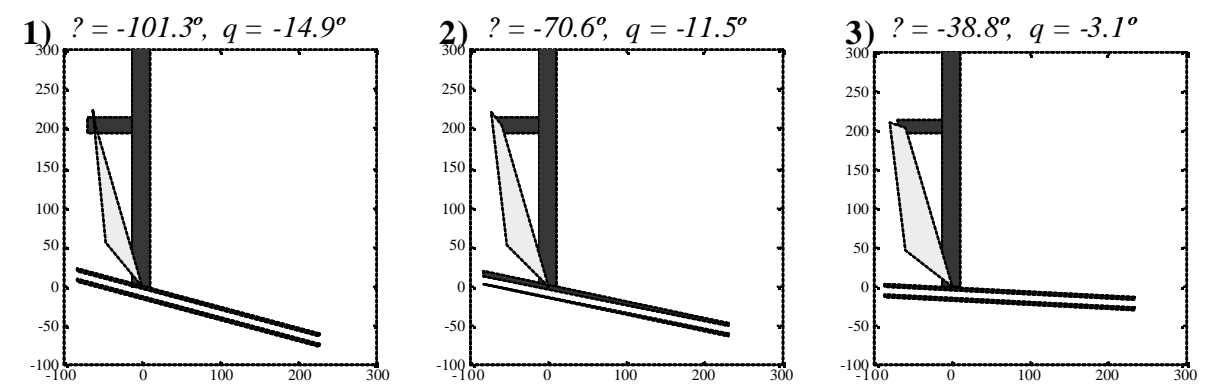

b)
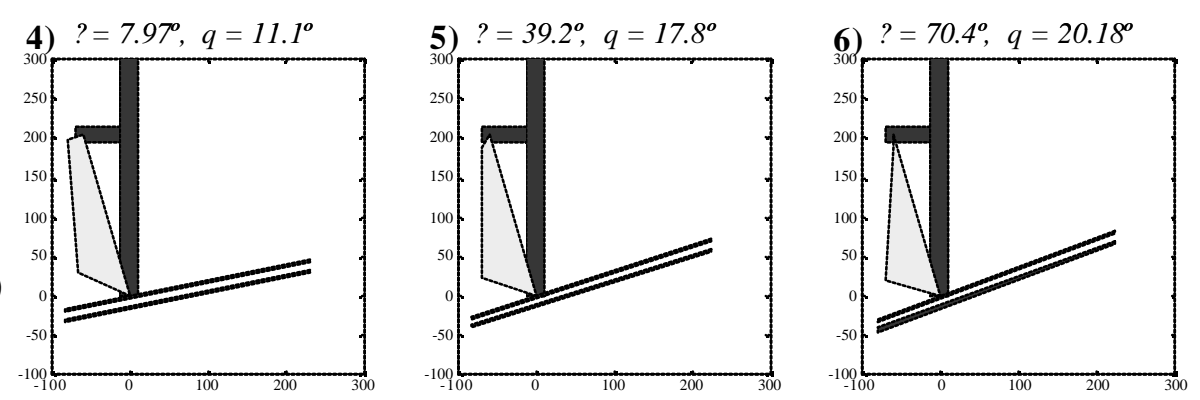

Figure 2 a) Non-linear relationship of SMART between input angle, output angle and transmission ratio; b) Foot trajectory according to MATLAB ${ }^{\circledR}$ simulation 
Some simulations in MATLAB ${ }^{\circledR}$ have been realised in order to choose the trajectory for the experiments (see Figure 2b). In these simulations, the foot trajectory was realised between the end positions of the transmission ratio function. These end positions take place when the crank and the rod are aligned. At these positions the output angle value is $-14.9^{\circ}$ or $20.18^{\circ}$, respectively. In Figure 2a, the configurations of the six frames of the simulations are discernible with black dots.

\section{SILO2 HUMANOID ROBOT GENERAL CONFIGURATION}

SILO2 is a humanoid robot designed at the Industrial Automation Institute with an initial number of 23 degrees of freedom. This design considers two degrees of freedom in each ankle, one in each knee, three in each hip, two in the trunk, three in each arm, and three in the head with stereo vision (Armada et al., 2003).

This humanoid robot has two different types of actuators: classical and non-linear actuators. Non-linear actuators drive two ankle joints in the sagittal plane, two knee joints in the sagittal plane, and two hip joints in the lateral plane (see Plate 1).

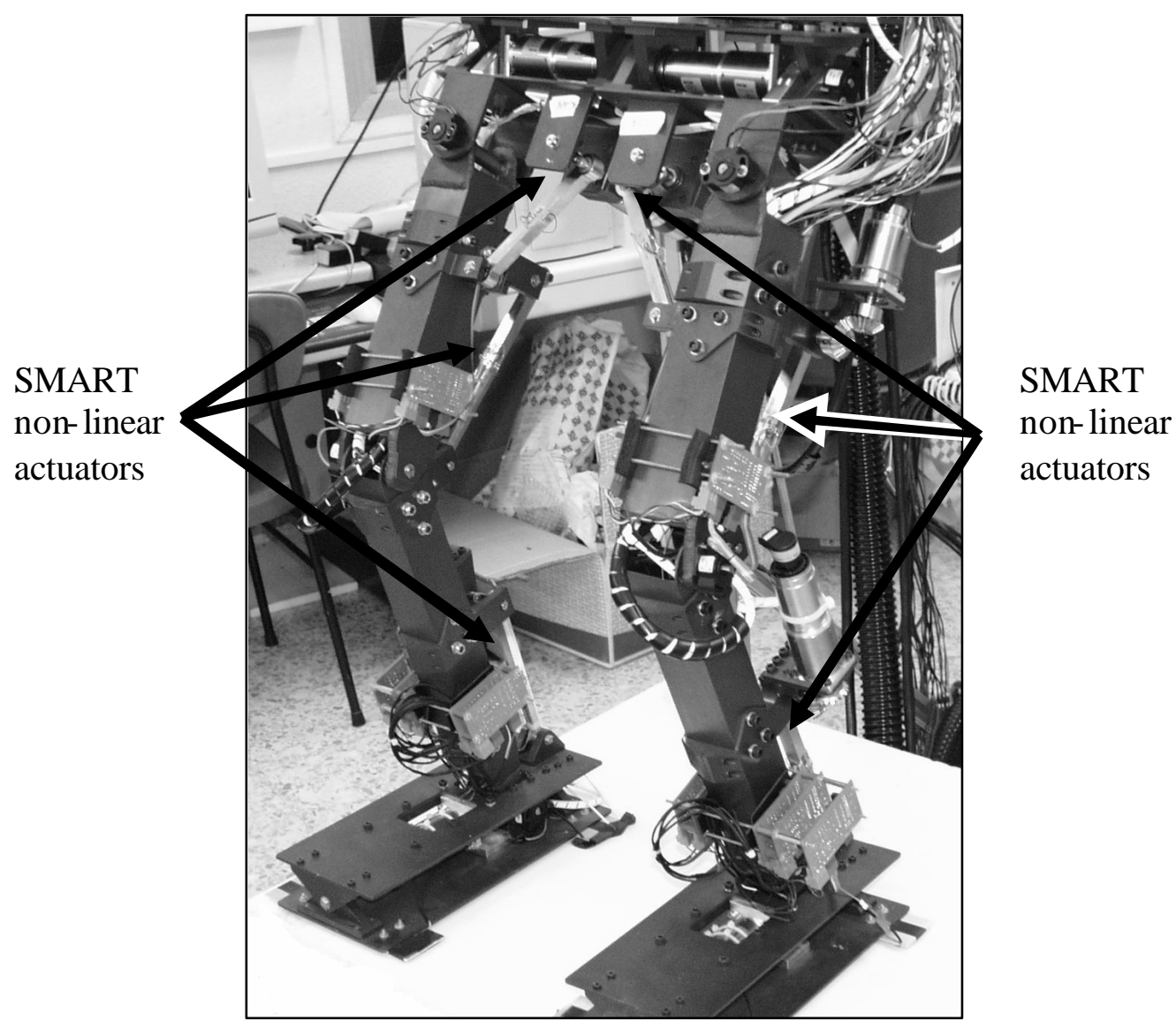

Plate 1 SMART non-linear actuators in SILO2

The sensorial system of SILO2 consists in: 23 incremental optical encoder (IE) placed in each motor's rotor axis, 23 rotary position sensor (these sensors are very important due to 
non- linear characteristic of the SMART actuator), 23 current sensor (which allow to supervise the effective power consumption on each motor), 6 force sensor (one placed on each SMART rod), 4 force sensor placed in each robot foot, 3 accelerometer in each foot and 3 more in the hip, 2 gyro rate in each foot, and two CCD 1/4" colour cameras.

Figure 3 shows a block diagram illustrating the multi-sensor system architecture (Pedraza et al., 2003). It can be noticed the modularity in the selected approach, which will permit an easy expansion of the present capabilities. The hardware for the data acquisition and control consists of master/server single board computer Pentium III $700 \mathrm{MHz}$ with $23 \mathrm{PID} / \mathrm{slave}$ processors. Also, there are 5 data acquisition boards with $16,12.5 \mathrm{KHz}$ effective A/D channels each of them, and 23, $20 \mathrm{KHz}$, PWM ports. All the software is running on Neutrino II real time operating system, which assures high levels of performance and reliability in this kind of application.

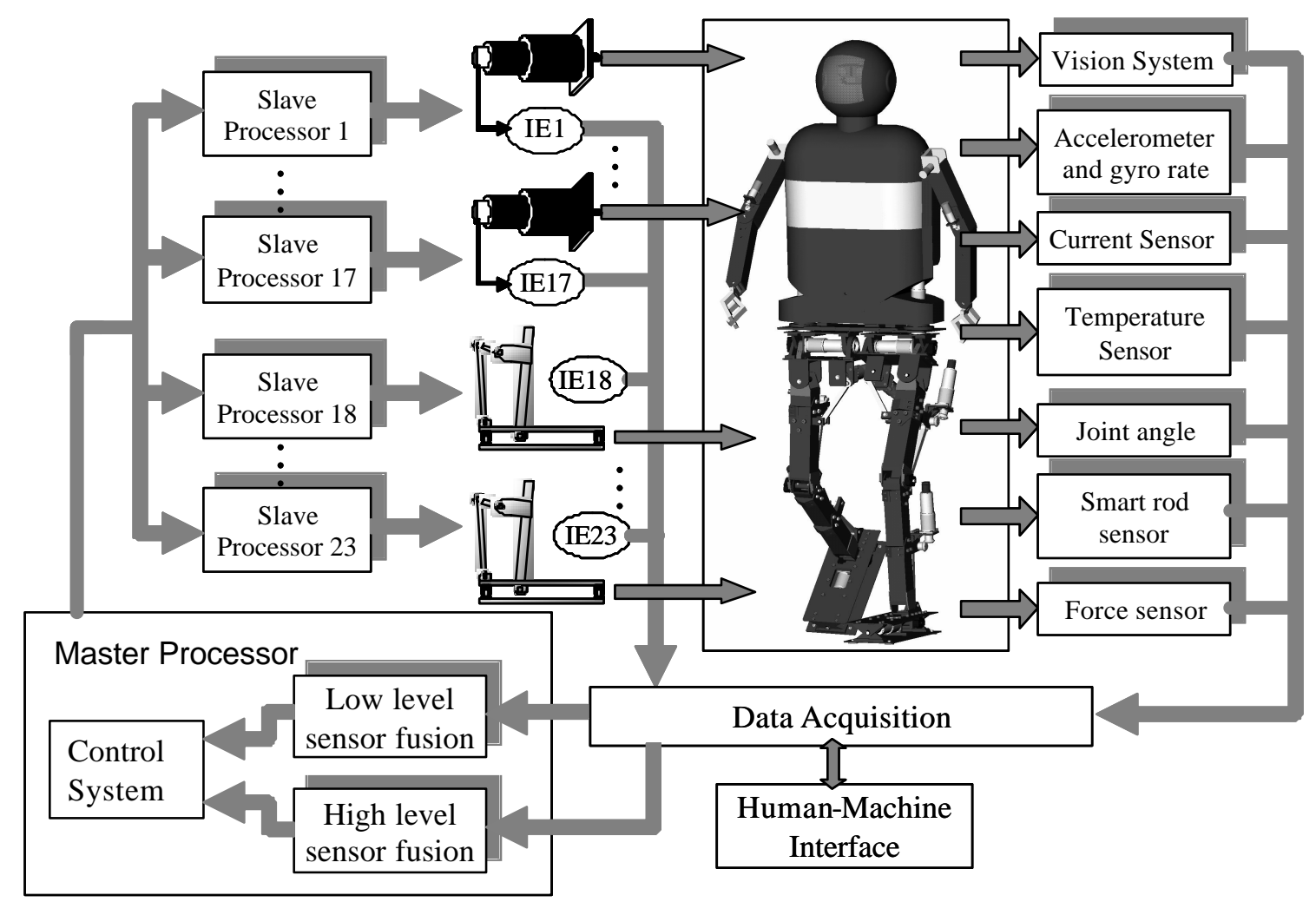

Figure 3 SILO2 system architecture

\section{ADDING EXTRA SENSITIVITY TO SMART BY FORCE SENSING}

The SMART non-linear actuator is inspired in the quasi-resonance drive (Akinfiev and Armada, 1998). Plate 2 shows one implementation of the SMART actuator, realised for driving the ankle of SILO 2 (Armada et al., 2002) humanoid robot. It can be noticed that the 
input angle (“??), driven by a dc motor with a planetary gearing, is measured using an optical encoder, and that the output angle (q) is measured using a precision potentiometer. The delivered torque to the output joint (q) is not directly available for measurement (no torque sensor exists) and, according to the theory, it is strongly correlated with the joint angular position, and it is non-linear. That is, the SMART mechanical transmission translates this load torque to the rotor of the driving motor (input angle, '?"') in a non-linear way determined by the four-linkage dimensions (please recall Figures 1 and 2), which results in an added difficulty.

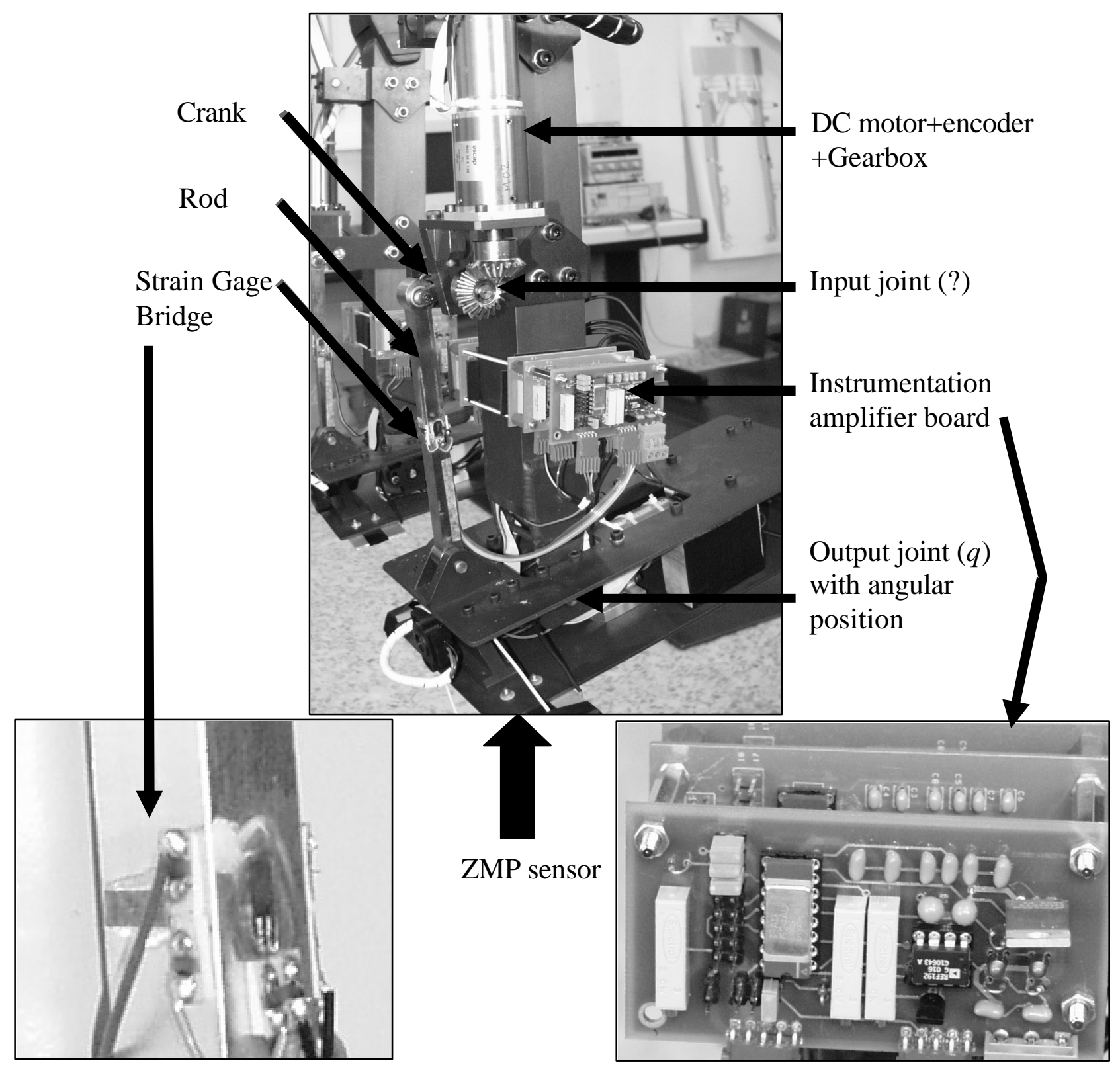

Plate 2 SMART non-linear actuator instrumented with added force sensor 
In previous works, the SMART actuator output joint (q) was controlled in position and speed with successful results (Caballero, 2002a). However, after many experiments and the use of 6 SMART actuators in a novel humanoid robot (SILO2) (see Plate 1) it was found (by several reasons concerning mainly observability and controllability) that it could be very interesting to enhance the responsiveness of SMART non-linear actuators (Montes et al., 2003) by adding new capabilities aimed to "measure" the output joint torque that, as it was explained before, is not directly available (this measurement cannot be implemented using off the shelf sensors because lack of space and cost). Because we are able only to measure the angles (and speeds) of the input/output joints, and the torque (throughout current measurement) of the input joint, it is needed to propose one method for the estimation of the output joint torque. So it was proposed to implement a force sensor in each one of the SMART actuators. This resulted in an improved responsiveness of this actuator, and will allow to implement an "indirect torque control" of the SMART output joint by using a force feedback control loop that uses the force sensor.

In all force sensors, forces and torques are measured through deformations or displacements in an elastic element of the sensor system (Gorinevsky et al., 1997). Strain gage transducers are the most extensively used for force measurement. In these transducers, strain causes a change in electrical resistance of strain gages mounted to an elastic element. There are several relatively techniques for mounting strain gages to the elastic element depending on the type of gage or applications. Strain gage force sensors have high sensitivity and measurement accuracy, and require relatively simple amplifiers. The main negative aspect of strain gages is their sensitivity to temperature, which has to be counteracted by special methods.

The placement of strain gages on one of the rods of the SMART actuator is employed to measure the force in the rod, and, subsequently, this measurement can be used to measure the torque at the output joint (and so the torque delivered to the external load). Plate 2 shows the modular electronic system and the strain gages. The strain gages are located like a classical pillar load cell. The force applied causes a compressive stress $-F / A$, where $A$ is the cross sectional area of the SMART rod. This loading conditions produces a longitudinal, compressive strain: $e_{L}=-F / A E$, which is accompanied by a transverse, tensile strain: $e_{T}=+v F / A E$, where $E$ and $v$ are respectively Young's modulus and Poisson's ratio for the rod material (Bentley 1995).

The strain gages are placed following a Wheatstone full bridge to convert the resistance output into a voltage signal. In this deflection bridge the resistances $R_{1}$ and $R_{3}$ are placed transversely on the SMART rod, while the resistances $\mathrm{R}_{2}$ and $\mathrm{R}_{4}$, are placed longitudinally on the rod; thus:

$$
R_{1}=R_{3}=R\left(1+\frac{G v F}{A E}\right) ; \quad R_{2}=R_{4}=R\left(1-\frac{G F}{A E}\right)
$$


Then, the output voltage of the deflection bridge is:

$$
V o=V s \frac{\frac{G F}{A E}(v+1)}{2+\frac{G F}{A E}(v-1)}
$$

Where, $G$ is the gage factor and $V s$ is supply voltage.

This output voltage is augmented with a modular instrumentation amplifier board of high precision, low noise, high gain accuracy, low gain temperature coefficient and high linearity designed for use with low level transducers, including load cells and strain gages.

This modular electronic board (see Plate 2) is used both to acquire the measurement from the load cells placed in each robot foot plant for ZMP estimation, and to get information from the strain gages placed on the SMART rod for indirectly output torque evaluation in the joint.

The forces acting on the rod of the non-linear actuator have been determined with the force sensor implementation, in order to calculate torque output in ankle joint, $\tau=F d \cos (\alpha)$ (see Figure 1). Torque output at ankle joint is then correlated with the torque on the electric motor and determined indirectly by measuring the supplied current. With both magnitudes the torque at the ankle joint can be estimated.

\section{SENSOR FUSION AND EXPERIMENTAL RESULTS}

As it was explained before the theoretical advantages of non-linear actuators has a major drawback in their innate complexity. To solve this problem it is required to have direct access to some variables, like the output torque, what is not possible by practical (mechanical design) and economical reasons (high cost). Under this conditions there is one alternative, to build-up an estimation of the essential variables by means of using the available sensors and performing some calculations. In other words, the idea is to use an approach based on sensor fusion.

The proposed sensor fusion strategy has been organised in two consecutive stages (please refer to Figure 4). The first stage is double: Fusion 1 uses motor current and output joint angle sensors to produce an estimation (fusion) of the required input torque; Fusion 2 uses output joint angle and rod force sensor to produce an estimation (fusion) of the output joint delivered torque. The second stage of sensor fusion (Fusion 3) is realised in an upper level and merges the estimated input and output joint torques to produce an estimation (fusion) of the nonlinear transmission ratio function.

For the sake of simplicity the experiments were carried out with the force sensor implemented in the rod of the SMART actuator driving the ankle of SILO2 humanoid robot. Many experiments were carried out and real time data associated with the measurement of angles at the output joint, current at the driving motor and the force in the SMART rod were recorded. On these data the sensor fusion strategy was implemented. Experimental results are illustrated 
in Figure 4, where it is possible to notice (central blocks) the relationship between motor torque and output joint torque along the output joint angle. The maximum motor torque happens when the output angle is near to $5^{\circ}$. At this time, the crank (see for illustration Figures 1,2 and 4) is at a 90 degree angle with the leg and the transmission ratio is the least possible one. The minimum motor torque comes about in the extremes of the trajectory, when the output angle gets the values $-14^{\circ}$ or $20^{\circ}$ (experimental values) and, in this case the transmission ratio of the four-bar mechanism tends to infinite. On the other hand, the maximum torque at the output joint happens when the foot is at a right angle to the leg, so near $\mathrm{q}=0^{\circ}$. From the central blocks of Figure 4 it is clear that both maximum torques are not in phase due to the non- linear characteristic of the SMART actuator.

The left side of Figure 4 shows the raw data and the filtered results corresponding to the measurements of the motor current, output joint angle and force measurement. It is to be noticed also that the output torque (see Figure 4, lower graphic, central block), that should be theoretically sinusoidal, do not follows exactly this assumption, mainly due to the natural delay involved with the filtering, and with the filtering itself.

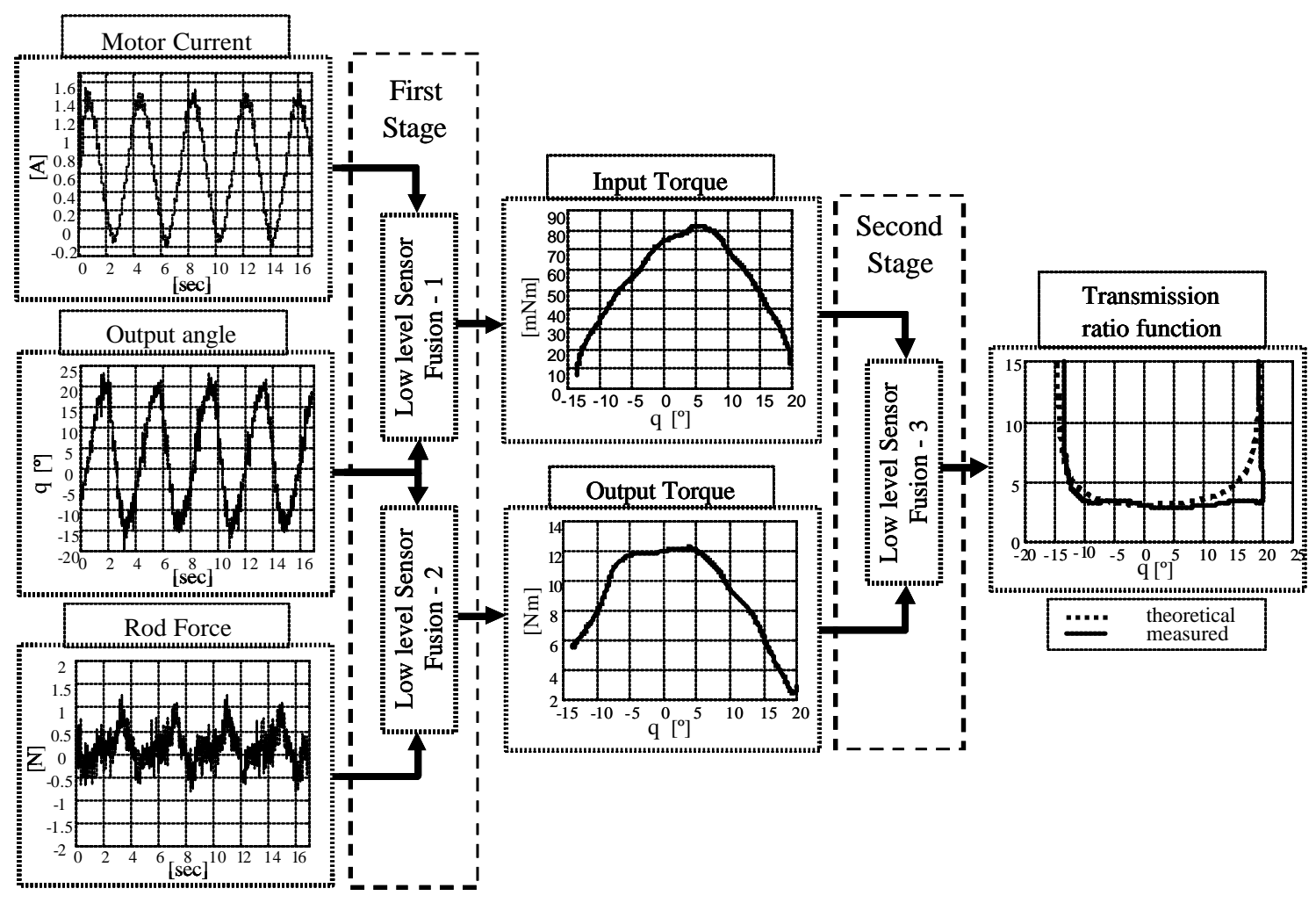

Figure 4 Two-stages sensor fusion strategy 
These experiments were realized with one mass of $5.84 \mathrm{~kg}$ placed over the foot end, at 185 $\mathrm{mm}$. of the ankle joint (see Plate 3). Also, it needs to be taken into account that the foot mass is about $2.25 \mathrm{~kg}$ with its centre of gravity located $79 \mathrm{~mm}$. from the ankle joint.

As a consequence of the previous results, it is clear that, for practical purposes, the instrumentation of a force sensor in one of the links of the SMART actuator enabled the indirect measurement, with good accuracy and repeatability of the torque delivered at the output joint.

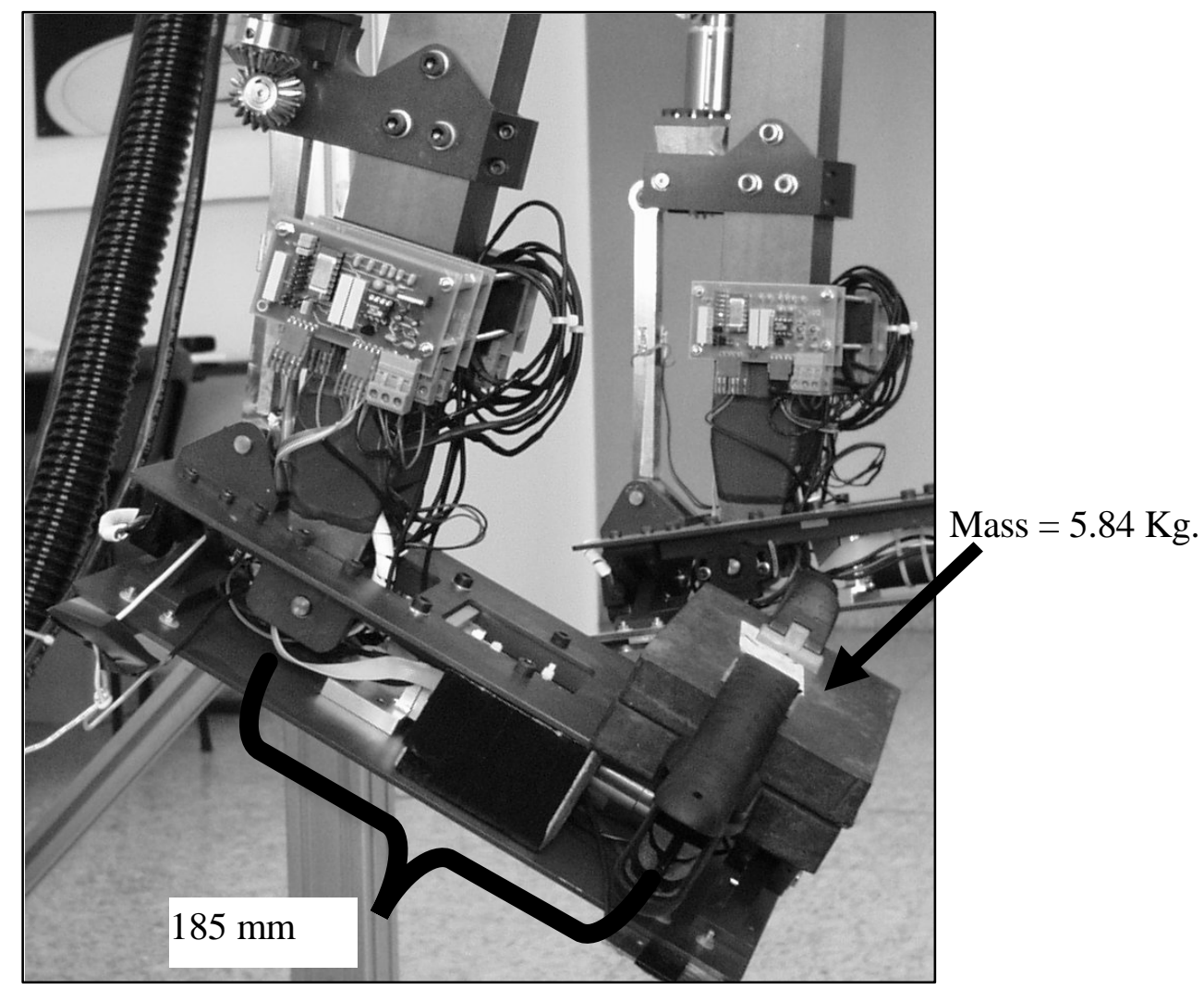

Plate 3 SILO2 foot loaded with $5.84 \mathrm{Kg}$.

The next step is to correlate the torque at the output joint (that is not powered) and the input motor torque (the only control element we have), in order to get a final assessment of the SMART actuator (see Figure 4). The experimental input/output relationship of the SMART drive can be obtained by dividing the respective (estimated) output and input torques. This leads to an experimental characterisation of the i/o relationship (the transmission ratio of this actuator).

The right block of Figure 4 (please recall here also Figure 2a) shows a comparison between the theoretical and the measured transmission ratio. It can be noticed that the obtained experimental reduction ratio is very high at the edges of the graphic, as it was expected. However, at the central part of the graphic, the dynamic effects become visible at the 
beginning of the trajectory, immediately after the high reduction ratio. This happens when the foot is starting to ascend (see Figure 2b). Also it is noticeable a backlash effect (due to the pinion gears) that appears at the end of the trajectory, when the foot is reaching its end (upper) position. The experiments have been performed many times with similar results.

One further type of experiment was aimed to the comparison of the power consumption between the SMART drive and a classical drive under the same working conditions in quasistatic movement (see Plate 4). In this experiment, the initial velocity was zero at the beginning of the trajectory (output angle $=-14^{\circ}$ ) and, also, the final velocity was zero (output angle $=20^{\circ}$ ).

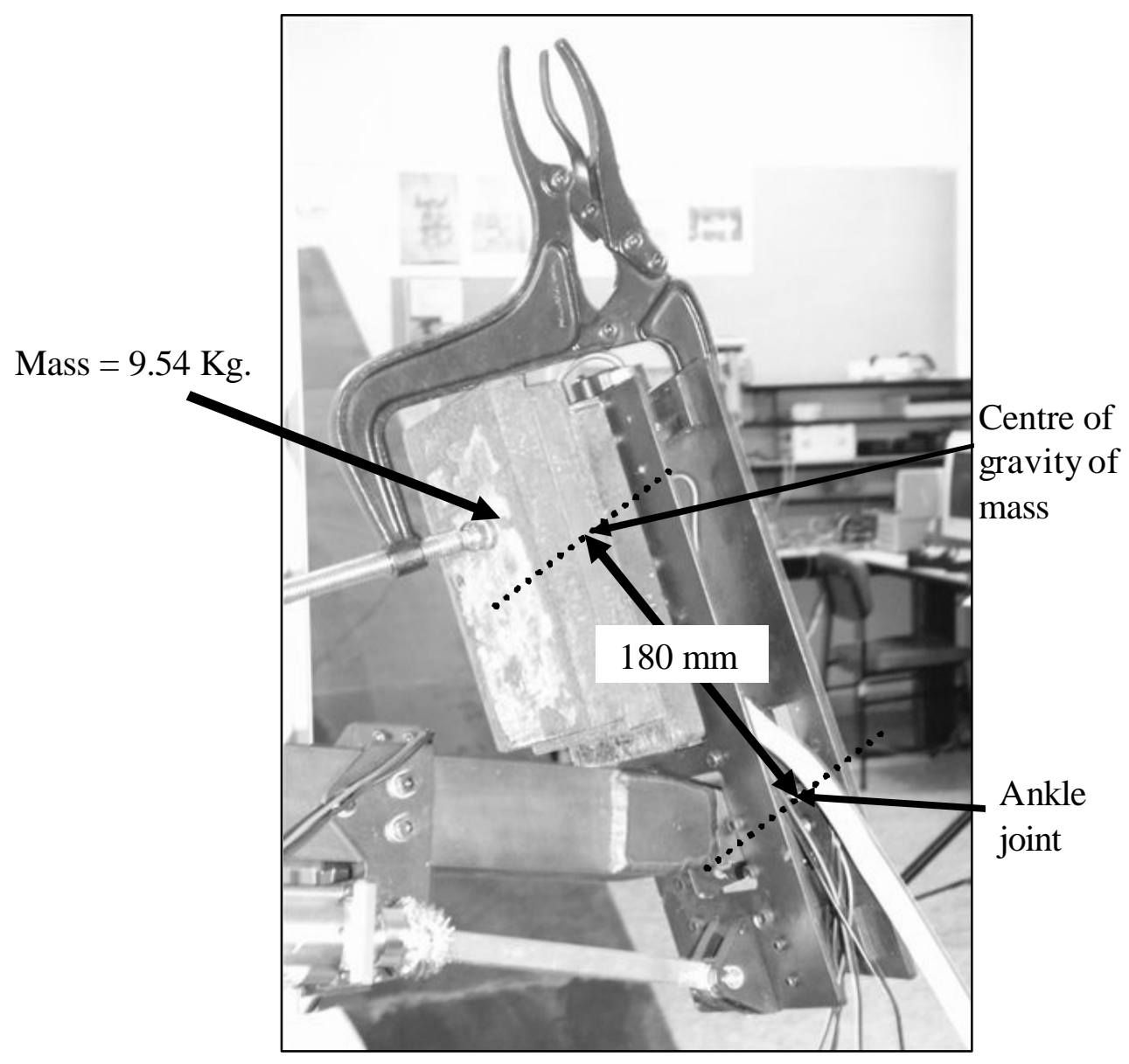

Plate 4 SILO2 Foot loaded vertically to comparison with classical transmission

It has been fully tested that with the SMART non-linear actuator the average electrical power consumption was of approximately 3.3 watts and with the classical actuator the average electrical power consumption was 6.4 watts (see Figure 5). This gives an average saving of energy of $48 \%$ of the SMART actuator in comparison with the classical drive. Especially interesting case can be noticed at the end positions of the trajectory, where SMART actuator clearly exhibits zero energy consumption (because of its infinitum transmission ratio and self braking properties), while the classical actuator is expending energy because the output torque is increasing. The Figure 5 shows absolute values of electrical power. 


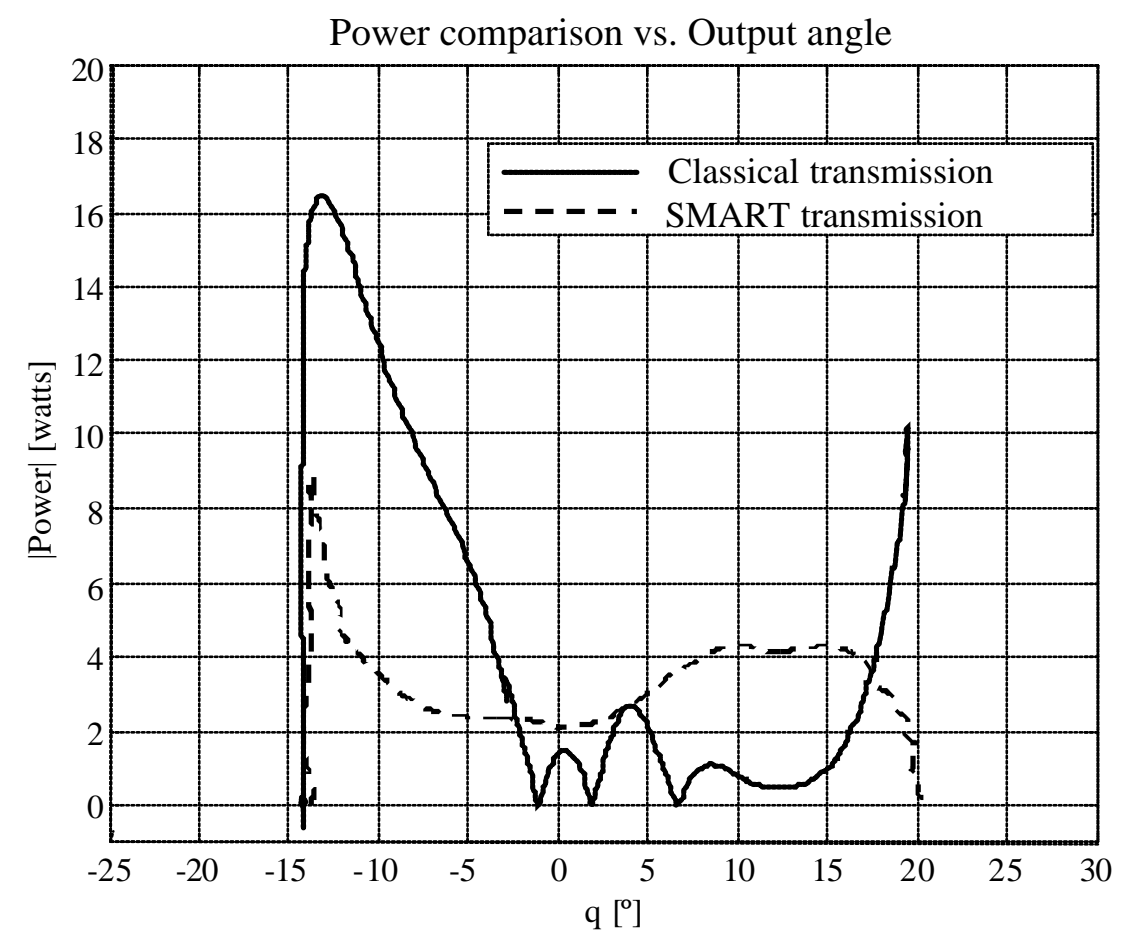

Figure 5 Power consumption comparison between classical and SMART actuator

\section{CONCLUSIONS}

The highly non-linear characteristic of SMART actuator offers some advantages for driving biped robots, but, on the other hand, their use implies some added complexity. With the final goal of enhancing the SMART responsiveness, it has been shown that a sensor fusion strategy where the main element consists in the implementation of a force sensor on one of the rods of the non-linear transmission provides an extra sensitivity and allows performing real-time, good quality measurement, of the output torque. So, the sensor fusion strategy has permitted the full testing of the foreseen SMART actuator properties and the indirect output joint torque monitoring throughout the evaluation of the force exerted on the rod along with the other available sensor data. A modular electronics has been used both to acquire the measurement from the load cells placed in each robot foot for the ZMP estimation and to get the signals from the strain gages. Also, the sensor fusion strategy has been proven very efficient and reliable to demonstrate that there is a fine correspondence between the theoretical transmission ratio function of the SMART drive and the experimental results. Therefore, with 
the addition of the sensor fusion, the SMART properties are now more easily understandable and useful. In addition, the experiments have shown that energy consumption decreases (48\% approximately) with the use of SMART non-linear actuator when compared to a classical constant transmission ratio actuator.

The obtained results are part of an on-going research and bring in new ideas (and tools) for improving the performance of biped locomotion and other related mechatronic devices.

\section{ACKNOWLEDGMENTS}

The authors would like to acknowledge the funding from Consejeria de Educacion y Cultura of Comunidad de Madrid, under research projects 07T/0040/1998 and 07T/0022/2001. The authors would like to recognise the help of Miss Veronica Garcia by her cooperation in SILO2 manufacturing. CLAWAR Thematic Network, funded by the EC, has been very supportive of all our activities in climbing and walking robots.

\section{REFERENCES}

Akinfiev, T., Armada, M., and Caballero, R. (2000), Actuador para las piernas de un robot caminante. Spanish patent Application P200002423.

Akinfiev, T. and Armada, M. (1998), 'Resonance and quasi-resonance drive for start-stop regime'. Proceedings of the $6^{\text {th }}$ International Conference MECHATRONICS'98. Skovde, Sweden. Pergamon, pp. 91-96.

Armada, M., Caballero, R., Akinfiev, T., Montes, H., Manzano, C., Pedraza, L., Ros, R., and Gonzalez de Santos, P. (2002), 'Design of SILO2 Humanoid Robot'. Proceedings of IARP Workshop on Humanoid and Friendly Robotics, December 11-12, Tsukuba, Japan, pp. 37-42.

Armada, M., Caballero, R., Akinfiev, T., Montes, H., and Pedraza, L. (2003), 'Extending Humanoid Robot functioning by proficient application of non-linear actuator'. Proceedings $11^{\text {th }}$ International Conference on Advanced Robotics, ICAR 2003. University of Coimbra, Portugal, June 30 - July 3, Vol. 3, pp. 1757-1762.

Bentley, J. P. (1995), Principles of Measurement Systems. 3rd ed. Harlow, England, Pearson Education Limited

Caballero, R. (2002a), Control of non-linearly actuated biped robots. Thesis (PhD). Polytechnic University of Madrid.

Caballero, R., Akinfiev, T., Montes, H., Manzano, C., and Armada, M. (2002b), 'Design of the SMART actuated ROBICAM biped robot'. Proceedings of International Conference of Climbing and Walking Robots 2002, September 25-27, 2002, Paris, France, pp. 409-416.

Gorinevsky, D. M. , Formalsky, A. M., and Schneider, A. Y. (1997), Force Control of Robotics Systems. Florida, CRC Press LLC 
McGeer, T. (1990), "Passive dynamic walking", International journal of Robotic Research $9(2), 62-81$

Mennito, G., and Buehler, M. (1997), "LADD Transmissions: Design Manufacture, and New Compliance Models", ASME Journal of Mechanical Design, 119(2), pp. 197-203.

Montes, H., Pedraza, L., Armada, M., Caballero, R., and Akinfiev, T. (2003), 'Force sensor implementation for enhanced responsiveness of SMART non-linear actuators'. Proceedings of International Conference on Climbing and Walking Robots, September 17-19, Catania, Italy, pp. 887-894.

Pedraza, L., Montes, H., Caballero, R., and Armada, M. (2003), 'Real Time Multi-Sensor Monitoring System for SILO2'. Proceedings of International Conference on Climbing and Walking Robots, September 17-19, Catania, Italy, pp. 975-982.

Pfeiffer, F., Löffler, K., and Gienger, M. (2000), 'Design Aspects of Walking Machines'. Proceedings $3^{\text {rd }}$ International Conference on Climbing and Walking Robots, October 2-4, Madrid, Spain, pp. 17-38.

Pratt, G., and Williamson, M. (1997), Elastic Actuator for Precise Force Control. US Patent: US5650704.

Van De Straete, H. and Schutter, J. (1999), 'Optimal time varying transmission for servo motor drives'. Proceedings of the Tenth World Congress on Theory of Machines and Mechanisms. Edited by Oulu University Press, Vol. 5, pp. 2055-2062.

Waldron, K. J. and Kinzel, G. L. (1999), Kinematics, Dynamics, and Design of Machinery. New York, John Wiley \& Sons, Inc

Yamaguchi, J. and Takanishi, A. (1997), 'Development of a Biped Walking Robot having antagonistic driven joints using nonlinear spring mechanism'. Proceedings of the 1997 IEEE International Conference on Robotics and Automation. Albuquerque, New Mexico.

\section{LIST OF FIGURE CAPTIONS}

Figure 1. SMART actuator: four-bar linkage and kinematics parameters

Figure 2. a) Non-linear relationship of SMART between input angle, output angle and transmission ratio; b) Foot trajectory in Matlab ${ }^{\circledR}$ simulation.

Figure 3. SILO2 system architecture.

Figure 4. Two-stages sensor fusion strategy.

Figure 5. Power comparison between classical and SMART actuator. 


\section{LIST OF PLATE CAPTIONS}

Plate 1. SMART non-linear actuators in SILO2

Plate 2. SMART non-linear actuator instrumented with added force sensor

Plate 3. SILO2 foot loaded with $5.84 \mathrm{Kg}$.

Plate 4. SILO2 Foot loaded vertically to comparison with classical transmission 